\title{
Vertical handover algorithm based on multi-attribute and neural network in heterogeneous integrated network
}

\author{
Xiaonan Tan ${ }^{1}$, Geng Chen ${ }^{1 *}$ (D) and Hongyu Sun ${ }^{1,2}$
}

\author{
*Correspondence: gengchen@ \\ sdust.edu.cn \\ ${ }^{1}$ College of Electronic and \\ Information Engineering, Shandong \\ University of Science and \\ Technology, Qingdao 266590, China \\ Full list of author information is \\ available at the end of the article
}

\begin{abstract}
A novel vertical handover algorithm based on multi-attribute and neural network for heterogeneous integrated network is proposed in this paper. The whole frame of the algorithm is constructed by setting the network environment in which we use the network resources by switching between UMTS, GPRS, WLAN, 4G, and 5G. Each network build their own three-layer BP (Back Propagation, BP) neural network model and then the maximum transmission rate, minimum delay, SINR (signal to interference and noise ratio, SINR), bit error rate, user moving speed, and packet loss rate which can affect the overall performance of the wireless network are employed as reference objects to participate in the setting of BP neural network input layer neurons and the training and learning process of subsequent neural network data. Finally, the network download rate is adopted as prediction target to evaluate performance on the five wireless networks and then the vertical handover algorithm will select the right wireless network to perform vertical handover decision. The simulation results on MATLAB platform show that the vertical handover algorithm designed in this paper has a handover success rate up to $90 \%$ and realizes efficient handover and seamless connectivity between multi-heterogeneous networks.
\end{abstract}

Keywords: Neural network, Multi-attribute, Vertical handover, Network selection

\section{Methods/experimental}

In this paper, in order to solve the problem of users switching networks under the condition of heterogeneous integrated network, we propose a vertical handover algorithm of heterogeneous integrated network based on neural network framework. We introduce the BP neural network to participate in the construction and execution of this algorithm, and introduce the $5 \mathrm{G}$ network in the environment where UMTS, GPRS, WLAN, and 4G networks coexist to improve the scope of this algorithm. The type of three-layer BP neural network uses user moving speed, maximum transmission rate, minimum transmission delay, signal-to-interference plus noise ratio, bit error rate, and packet loss rate as inputs of the neural network in a heterogeneous network environment. The six network input factors are set the middle layer of the neural network according to the relevant empirical formula, we use the network download rate as the

(c) The Author(s). 2020 Open Access This article is licensed under a Creative Commons Attribution 4.0 International License, which permits use, sharing, adaptation, distribution and reproduction in any medium or format, as long as you give appropriate credit to the original author(s) and the source, provide a link to the Creative Commons licence, and indicate if changes were made. The images or other third party material in this article are included in the article's Creative Commons licence, unless indicated otherwise in a credit line to the material. If material is not included in the article's Creative Commons licence and your intended use is not permitted by statutory regulation or exceeds the permitted use, you will need to obtain permission directly from the copyright holder. To view a copy of this licence, visit http://creativecommons.org/licenses/by/4.0/. 
key factor to determine the performance of the current wireless network, and then set it as the output of the neural network to participate in the vertical handover decision. Compared with other intelligent algorithms represented by fuzzy logic algorithms, the vertical handover algorithm improves the success rate of network handover and makes the seamless handover between heterogeneous networks more efficient.

\section{Introduction}

In recent years, with the rapid development of wireless network technology and the rapid advancement of network access technology of the heterogeneous convergence architecture, many different types of wireless networks exist simultaneously [1, 2]. Different networks compete and complement with each other, resulting in the coexistence of multiple types of network [3, 4]. In order to adapt to the different requirements of users and the development of various types of business, wireless networks with different architectures form a multiple heterogeneous integrated network [5-7], in which the most representative is the integration of two kinds of heterogeneous networks: WLAN and cellular [8-11]. WLAN networks have relatively wide bandwidth and small coverage, while cellular series networks (such as current 3G/4G wireless networks) have relatively narrow bandwidth and wide coverage [12-14], so the integration of cellular series network and WLAN network can complement each other [15, 16], giving full play to their respective strengths and restraining their limitations at the same time [17-19], in addition, with the birth of $5 \mathrm{G}$ technology, the integration between multiple networks with different architectures will make a revolutionary progress [20-25]. Therefore, the vertical handover algorithm should be considered with a variety of disturbing factors and a variety of candidate network types [26], and it is more suitable to be combined with intelligent algorithms [27]. The relevant neural network algorithms in the field of machine learning are now used in the design of this paper's algorithm [28-32].

There are many vertical handover algorithms, and each has its own advantages and disadvantages. Reference [1] proposed a vertical handover algorithm based on visible light communication system for decision selection. By utilizing analytic hierarchy process and a two-person cooperative game model to consider multiple attributes, including dynamic network parameters and actual traffic preferences. Although handover was reduced. Overhead, it considered fewer handover factors. Reference [3] proposed a vertical handover decision algorithm for cooperation of multi-terminal based on fuzzy logic and analytic hierarchy process. Although it could fully reflect the application requirements and user preferences, it lacked consideration of the upcoming 5G network. Similar algorithms were mentioned in the reference [7, 17]. Reference [4] reviewed and classified the most significant multi-criteria decision-making algorithm for network selection in next-generation wireless networks, and illustrated an insight into the importance of network-criteria weighting and the current research trended in the application of multi-criteria decision-making algorithms to network-selection problems in heterogeneous wireless networks. Reference [6] proposed to maximize network utilization as more than one target network existed during vertical handover. Also, they showed how network parameters could be embedded into IEEE 802.21-based signaling procedures to provide seamless connectivity during a handover. However, the algorithm lacked consideration of the important factor of network transmission rate. The vertical handover algorithm proposed in reference [8] mainly used "New Composite Rule Inference 
based logarithm" to improve the service quality between WLAN_UMTS (Wireless Local Area Network and Universal Mobile Telephone System) networks. Although it had a high handover success rate, it also did not integrate the 4G and 5G network. Reference [9] was similar to reference [3], reference [7], and reference [8]. It employed fuzzy logic theory and multi-attributes decision making in selecting the best network to optimize the vertical handover algorithm, but did not involve the network attribute of SINR. Reference [11] proposed an adaptive vertical handover algorithm based on artificial neural network similar to the algorithm designed in this paper, but it only chose the LTE and WLAN network as candidate network. Reference [12] presented a mixed integer linear programming (MILP) model to balance multi-homing loads in heterogeneous wireless networks based on multi-objective tabu search method. Both the reference [13] and the reference [15] applied the genetic algorithm to the relevant handover decision, which had a high success rate of handover, but also lacked consideration of 5G network factors. Reference [16] proposed a heterogeneous network selection algorithm based on the combination of network attributes and user preferences, similar to the multi-attribute-based vertical handover algorithm. Reference [20] designed an intelligent algorithm for Seamless Vertical Handover in vehicular networks, which were essentially based on a hybrid model merged the biogeography-based optimization or BBO with the Markov chain, and applied to the vertical handover between different access technologies such as WiMAX, WiFi, and UMTS. However, this handover process was more complicated. Reference [18], reference [22], and reference [24] emphasized the application of 5G network technology, but failed to integrate perfectly with vertical handover algorithms in heterogeneous networks. Reference [26] considered factors related to inter-cell interference in communication activities, but the related theory was more complicated. Reference [27] introduced fuzzy logic algorithm into wireless positioning, and failed to combine related network environment factors. The literature [2832] generally used the relevant neural network algorithms in the field of machine learning, but the theories mentioned had been improved to a certain extent. Although the efficiency was improved, the operation process of the algorithm was also more complicated .

In summary, the environmental factors that can affect the performance of wireless networks considered by most of the current vertical handover algorithms in the engineering field are not comprehensive. With the 5G network about to popularize, most existing vertical handover algorithms affect the performance of $5 \mathrm{G}$ networks. But the related factors of $5 \mathrm{G}$ networks are not included, which causes most of the existing vertical handover algorithms will not be able to switch to the $5 \mathrm{G}$ network when selecting the network in the area covered by the $5 \mathrm{G}$ network.

Therefore, to solve the drawback of existing vertical handover algorithm including the high complexity of the running process and exclude $5 \mathrm{G}$ network system, a novel vertical handover algorithm based on multi-attribute and neural network for heterogeneous integrated network is proposed in this paper. The whole frame of the algorithm is constructed by setting the network environment in which we use the network resources by switching between UMTS, GPRS, WLAN, 4G, and 5G. Each network build their own three-layer BP (Back Propagation, BP) neural network model and then the maximum transmission rate, minimum delay, SINR (signal to interference and noise ratio, SINR ), bit error rate, user moving speed, and packet loss rate which can affect the 
overall performance of the wireless network are employed as reference objects to participate in the setting of "BP neural network input layer neurons" and "the training and learning process of subsequent neural network data." Finally, to perform vertical handover decision, the network download rate is adopted as prediction target to evaluate performance on the five wireless networks and it will help the vertical handover algorithm designed in this paper select the right wireless network.

This vertical handover algorithm is applied to the network's selection with different architectures. Therefore, when performing relay selection between networks with different architectures, the six network characteristics set at the input of the neural network model corresponding to the above five networks are used to select to implement a decision strategy, that is, to predict the final download rate of the five networks based on the real six network attributes that have been input into the neural network model, so as to choose the existing five networks to be robust and optimal in the current environment. The network with the highest download rate to be selected as the final switch and gives users a good experience.

Compared with most of the existing vertical handover algorithms, the novelty of the vertical handover algorithm designed in this paper is to include the 5G network in the switching range of the heterogeneous integrated networks, the use of the excellent learning ability, and the independently designable framework integrates most of the key factors that affect the performance of the wireless network into this vertical handover algorithm. Therefore, the environmental factors related to the performance of the wireless network considered by the algorithm in this paper are more comprehensive.

This paper is organized as follows. First, the proposed methods are presented in Section 2. Simulation and experimental results are given in Section 3. Finally, the conclusion is presented in Section 4.

\section{Proposed method}

\subsection{Neural network model}

The vertical handover algorithm proposed in this paper is designed for UMTS, GPRS, WLAN, 4G and 5G networks. Each network build their own three-layer BP neural network models. The neuron weights of each layer are determined according to the theoretical values of the maximum transmission rate, minimum delay, SINR, bit error rate, user's moving speed, packet loss rate, and network download rate. When the user enters into the network environment where the five heterogeneous networks are coexisted, the terminal collects the actual maximum transmission rate, minimum delay, SINR, bit error rate, user moving speed, and packet loss rate according to the algorithm and inputs the values to the five neural network models that have been trained respectively. The predicted values of the download rates of network corresponding to each of the five types of networks are obtained, and finally the wireless network with highest network download rate prediction value is selected as the pre-switched network.

Since the vertical handover algorithm designed in this paper is based on the BP neural network model, Fig. 1. shows the generalized three-layer BP neural network model. 


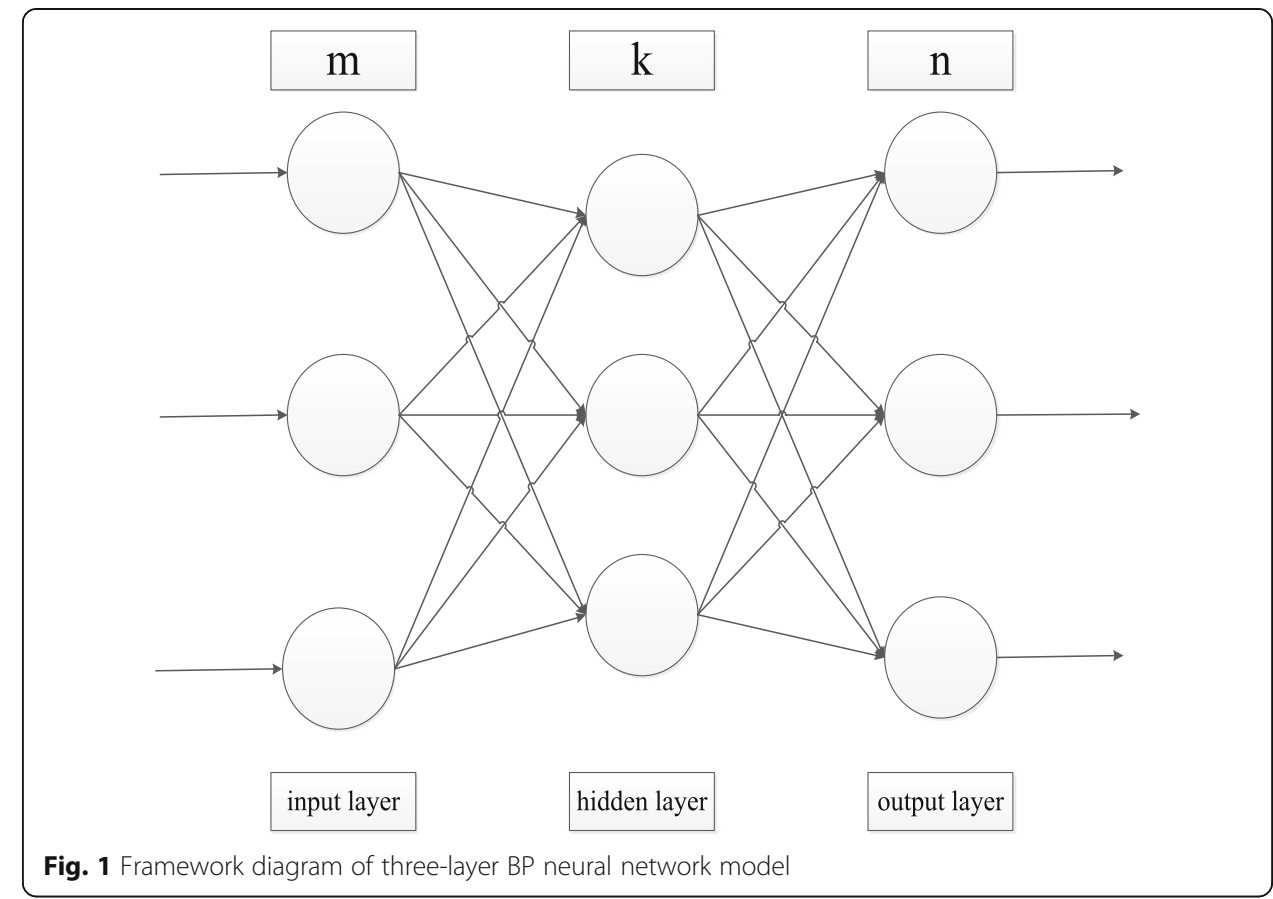

Where $m, k$, and $n$ represent the neurons in the input layer, hidden layer, and output layer, respectively. BP neural network model is constructed by using Log-sigmoid transfer function as Eq. (1).

$$
f(x)=\frac{1}{1+e^{-x}}
$$

At the same time, the error back propagation algorithm and the error function (2) are employed in this work, so that the weights value of the neural network are constantly regulated by the error feedback, and the offset value of the error function is more closer to the expected one.

$$
E=\frac{\sum_{i}\left(t_{i}+O_{i}\right)^{2}}{2}
$$

Where $t_{i}$ are the expected values of the network parameter results and $o_{i}$ denote the related output values calculated by the neural network, respectively. And the corresponding neural network model needs to be solved for the operation of the algorithm.

Therefore, the operating principle of the BP neural network model shown in Fig. 1 is given now, the network weights are now set to $\left(W_{\mathrm{ij}}, T_{\mathrm{ij}}\right)$, the neuron threshold is set to (a), $X_{\mathrm{j}}$ is the $j$ th input layer node, $Y_{\mathrm{j}}$ is the $j$ th hidden layer node, $O_{\mathrm{j}}$ is the $j$ th output layer node, and $E$ is the error function, then the relevant calculation formulas of the above three-layer BP neural network model are as follows, where the neurons are set the input vector is $X=\left(x_{1}, x_{2}, x_{3}, \ldots \ldots, x_{\mathrm{m}}\right)$, then the weights value corresponding to the input vector in the input neuron is $W=\left(w_{1}, w_{2}, w_{3}, \ldots . . ., w_{\mathrm{m}}\right)$, the output of the neural network is expressed by Eq. (3): 


$$
y=f\left(\sum_{i=1}^{\mathrm{m}} w_{i} x_{i}-a\right)
$$

where $f$ is the activation function and its expression is Eq. (4):

$$
f(x)=\left\{\begin{array}{cc}
1, & x \geq 0 \\
-1, & x<0
\end{array}\right.
$$

The output of the hidden layer node is Eq. (5):

$$
y_{i}=f\left(\sum_{j} w_{i j} x_{j}-a_{i}\right)=f\left(\text { net }_{i}\right)
$$

The output of the output layer node is Eq. (6):

$$
o_{l}=f\left(\sum_{j} T_{i j} x_{j}-a_{l}\right)=f\left(\text { net }_{l}\right)
$$

Then, the error of the output layer node is Eq. (7):

$$
E=\frac{1}{2} \sum_{l}\left(t_{l}-o_{l}\right)
$$

Therefore, minimizing the error $E$ is the ultimate goal of the BP neural network for training and learning, so the weight adjustment of the BP neural network and the negative gradient of the error $E$ should be in a proportional relationship. It is expressed as Eq. (8).

$$
\frac{\partial E}{\partial T_{l i}}=\sum_{k=1}^{m} \frac{\partial E}{\partial o_{k}} \frac{\partial o_{k}}{\partial T_{l i}}=\frac{\partial E}{\partial o_{l}} \frac{\partial o_{l}}{\partial T_{l i}}
$$

The gradient of the error function of the hidden layer node is Eq. (9):

$$
\frac{\partial E}{\partial w_{l i}}=\sum_{l} \sum_{i} \frac{\partial E}{\partial o_{l}} \frac{\partial o_{l}}{\partial y_{i}} \frac{\partial y_{i}}{\partial w_{i j}}
$$

The gradient of the node error function of the output layer is Eq. (10):

$$
\frac{\partial E}{\partial a_{l}}=\sum_{k=1}^{m} \frac{\partial E}{\partial o_{k}} \frac{\partial o_{k}}{\partial a_{l}}=\frac{\partial E}{\partial o_{l}} \frac{\partial o_{l}}{\partial a_{l}}
$$

In summary, the BP neural network is a multi-layer feed-forward network trained according to error back propagation algorithm. In this algorithm, first, a little value is given to the connection value of network, and then, a training sample is selected to calculate gradient of error relative to this sample. By utilizing the methods of mean square error and gradient descent to realize the modification to the connection weights of network. The continuous modification of weight values and offset values are applied to make the real output of network more closer to the expected one.

Figure 2 shows the schematic diagram of the three-layer BP neural network model designed in this paper. (The five types of networks correspond to the five models respectively. Here we take the $5 \mathrm{G}$ network model for example). 


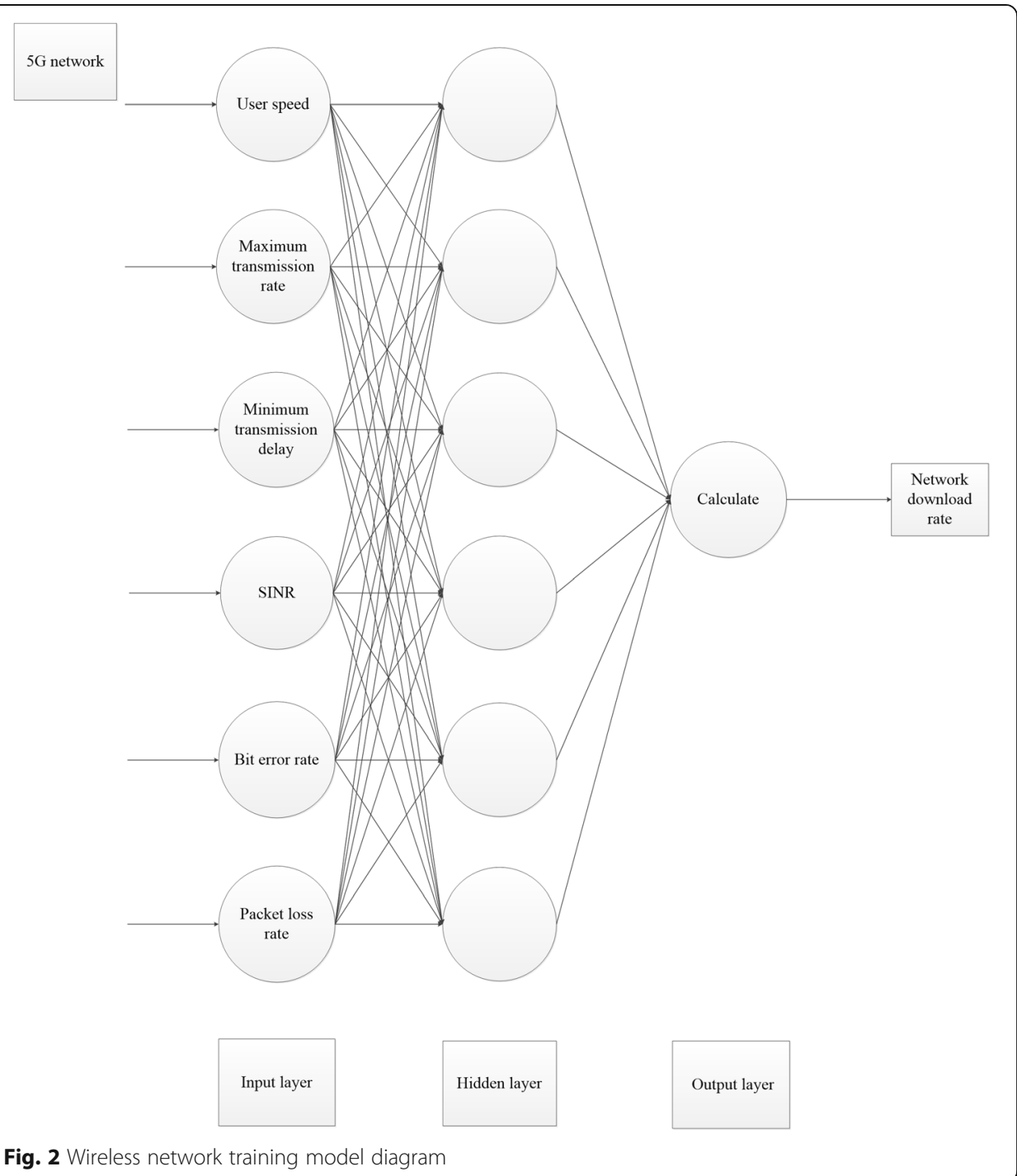

The neural network model construction of this work can be described as follows:

(1) First, the input layer of network and the attribute elements should be required. As presented above, the maximum transmission rate, minimum delay, SINR, bit error rate, user moving speed, and packet loss rate which can affect the overall performance of the wireless network are employed as reference objects to participate in setting of BP neural network input layer neurons and the training and learning process parameters of subsequent neural network data. In order to ensure that the designed algorithm utilizes multiple network attributes comprehensively and makes effective handover strategies, the network download rate is adopted as prediction target to evaluate the network environment on the five wireless networks in which the user is located and selects the right wireless network that will help the algorithm to perform the process of vertical handover. Therefore, six neuron nodes are set at the input layer of the respective BP neural network models of the five wireless networks, 
respectively. The six input layer neuron nodes respectively represent the maximum transmission rate, minimum delay, SINR, bit error rate, user moving speed, and packet loss rate corresponding to each wireless network in the heterogeneous integrated network composed of the five major wireless networks.

(2) To design the hidden layer. According to the empirical formula (11) of the number of hidden layer neurons to determine the number of hidden layer neurons:

$$
l=\sqrt{n+m+a}
$$

Where $n$ is the number of neural nodes in the input layer of network, $m$ is the number of neural nodes in the output layer of network, and $a$ denotes one number between 1 and 10 (including 1 and 10). And $l$ is the empirical value of the final number of hidden layer neuron nodes.

As the hidden layer of the BP neural network model, the neuron nodes of the hidden layer play an extremely important role. In theory, a considerable amount of neuron node of hidden layer can perform an infinite numerical approximation on a nonlinear function with arbitrarily small error precision. But the number of hidden layer neuron nodes should not be too large, otherwise it will increase the computational complexity and computational cost of the network. At the same time, complex network computing is very prone to over-fitting. Conversely, if the number of hidden layer neurons is very small, the error will increase, which will seriously affect the performance of the neural network, and thus result in excessively difference from the expected theoretical value of desired decision. Appropriate number of hidden nodes can lower computational complexity and improve the training convergence speed. There are considerable correlation among the number of hidden layer neuron nodes, the actual complexity of the heterogeneous integrated network, the number of neuron nodes in the input layer and the output layer, and the expected error values set by the vertical handover decision. Finally, after comprehensive consideration, during the data training of the neural network, based on the principle of minimizing the final value of the mean square error function of the BP neural network, six neurons are set in the hidden layer according to the formula (11).

(3) The third step is to design the output layer. Those six attribute data of five heterogeneous network collected by the terminal are finally input to their respective models, and then through network training, numerical approximation and prediction, and output their respective predictions of network download rate for the following decision.

Finally, after determining the prediction values of network download rate representing the five types of wireless networks and then comparing the value to select the most suitable wireless network at time. Therefore, for each neural network model corresponding to the wireless network, the number of neurons in output layer is set to one.

In summary, the three-layer BP neural network model corresponding to the five networks is built. Each model adopts the "6, 6, 1" mode, namely input layer have six neurons, hidden layer have six neurons, and output layer has one neuron, respectively. In the process of BP neural network training, for the maximum transmission rate, minimum delay, SINR, bit error rate, user moving speed, and packet loss rate of the five 
major wireless networks, take appropriate sample data and divide them into five groups as a reference sample of the BP neural network "6,6,1" model corresponding to each of the five wireless networks for instructor learning, then train the BP neural network models corresponding to the five wireless networks and after all of them have reached the corresponding mean square error standard, collect real data about the maximum transmission rate, minimum delay, SINR, bit error rate, user moving speed and packet loss rate in the heterogeneous integrated network environment and then input the real data of these six network attributes in the environment to the BP neural network "6,6, 1 " model corresponding to the five wireless networks that have been trained, the six BP neural network "6,6,1" models will obtain their respective network download rate prediction values through prediction. Then compare the six network download rate prediction values and finally select the wireless network corresponding to the highest value as the next network switching target, so that the algorithm completes a vertical handover process.

\subsection{Vertical handover process}

Based on the network model designed in Section 2.1, Fig. 3 shows the flow diagram of the implementation of the handover strategy designed in this paper.

The steps of the algorithm are the following:

(1). The vertical handover algorithm starts to run when the user terminal collects relevant network attribute parameter values in a heterogeneous converged network environment composed of five wireless networks.

(2). Next, the collected six types of network attribute parameters are sent to the input layer neurons of the three-layer BP neural network model represented by the five networks.

(3). For the network attribute values that have been input to the neural network model, the BP neural network performs numerical calculation and approximation according to the previously reference values and expected values, and finally find the internal correlation of the neural network corresponding to each wireless networks' weights.

(4). Then, according to the collected network attribute values, using the trained and learned BP neural network model to predict the network download rate of participating the final decision.

(5). Finally, based on the predicted network download rates of the five wireless networks that have been predicted, numerical comparisons are made, and based on the compared numerical results, the network with the best performance is selected to perform the vertical handover decision.

The above five steps are the required operation to perform a handover decision for the vertical handover algorithm designed in this paper. In the real environment where the five heterogeneous networks are integrated, when the user moves to a different location, the six network attribute parameters set in this paper will change with the network environment around the user. Therefore, the data need to be collected continuously to achieve network handover in a realistic heterogeneous converged network environment. That is, the above five steps need to be continuously cycled to complete multiple decisions to adapt to the dynamic network environment. 


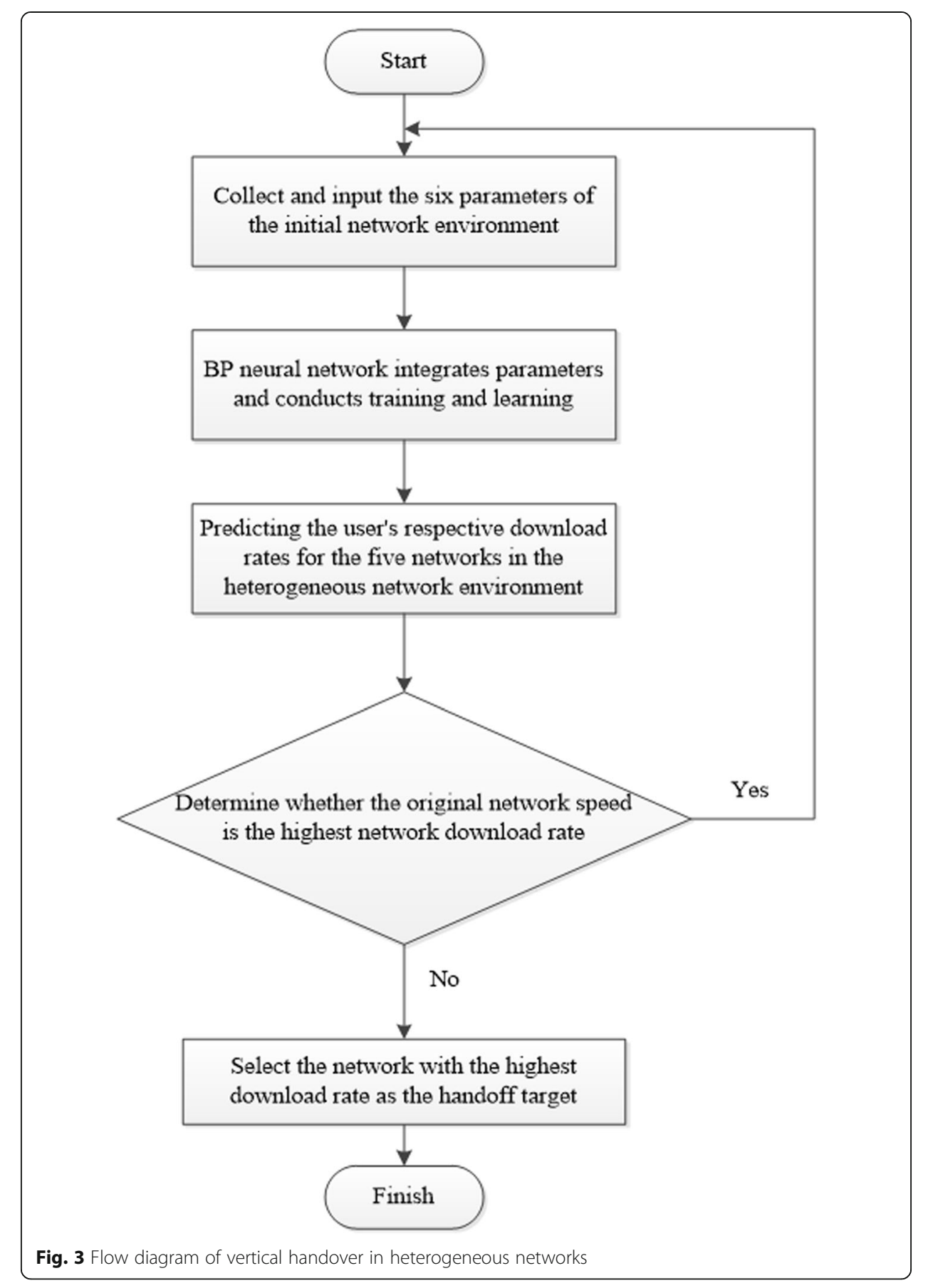

Table 1. Experience value of training parameters in heterogeneous integrated network

\begin{tabular}{llllllll}
\hline $\begin{array}{l}\text { Network } \\
\text { type }\end{array}$ & $\begin{array}{l}\text { User } \\
\text { speed(m/m/ } \\
\mathbf{s})\end{array}$ & $\begin{array}{l}\text { Maximum } \\
\text { transmission } \\
\text { rate (kbps) }\end{array}$ & $\begin{array}{l}\text { Minimum } \\
\text { transmission } \\
\text { delay }(\mathbf{m s})\end{array}$ & $\begin{array}{l}\text { SINR } \\
\text { Bit error rate } \\
\text { (the order of } \\
\mathbf{1 0} \text { ) }\end{array}$ & $\begin{array}{l}\text { Packet } \\
\text { loss } \\
\text { rate }\end{array}$ & $\begin{array}{l}\text { Network } \\
\text { download } \\
\text { rate (kbps) }\end{array}$ \\
\hline UMTS & 3 & 1024 & 20 & 18 & 3 & 0.005 & 2048 \\
GPRS & 3 & 115 & 30 & 16 & 10 & 0.009 & 300 \\
WLAN & 3 & 51200 & 7 & 22 & 7 & 0.002 & 102400 \\
$4 G$ & 3 & 20480 & 15 & 20 & 0.8 & 0.001 & 102400 \\
$5 G$ & 3 & 102400 & 1 & 25 & 0.01 & 0.0001 & 512000 \\
\hline
\end{tabular}




\section{Simulation results and analysis}

The algorithm uses the MATLAB R2016b software platform for simulation evaluation. Table 1 shows the empirical values of the network attributes of the five networks and uses those values as a benchmark to take a certain range of fluctuations for supervised neural network learning.

The following is the simulation steps of the BP neural network model in this paper:

(1). Firstly, the six network attribute reference values are normalized as neural network sample training parameters and input to the three-layer neural network model.

(2). At the same time with step (1), respectively set up excitation functions of the BP network between input layer and the hidden layer neural network structure and the output layer neural network structure as the "logsig" node transfer function and the "purelin" node transfer function for parameter transfer and network training.

(3). Then, set the initialization parameters inside the neural network, that is, a reasonable neural network iteration number(epochs) and predicted values expected error threshold (goal) are set for the algorithm.

(4). Here, the algorithm designed in this paper sets the network iteration training number to 5000 times, sets the target mean square error of the training network expected prediction value to 0.00065 , and sets the corresponding learning rate(lr) to 0.05 .

(5). After setting all the initial parameters, start training the network learning process and run the designed algorithm.

In summary, the algorithm designed in this paper is to set 20 sets of learning parameters for neural network training for each of the five networks, and then, we input the six network environment attribute values set according to the environment into their respective nerves. The handover process is run in the input layer of the network model, and finally the corresponding simulation results are obtained according to the selection of the performance optimal network by the neural network model.

Figures 4, 5, and 6's curves are BP neural network training error curves, training state curves, and data regression analysis curves, respectively. (Taking the training of related data of BP neural network model corresponding to UMTS network as an example).

The three solid lines shown in Fig. 4 are train, validation, and test, which represent the performance of the mean square error index of the BP training process in iteration, the performance of the mean square error index of the BP cross-validation process in iteration, the mean square error index of the BP testing process is expressed in iteration. The test line represents the BP calculation and the final result of the training. In addition, the best dotted line in the figure shows the algorithm designed in this paper when the neural network is trained to the seventh generation. The BP training result is the best, and the "goal dashed line" indicates the value of the network training termination target set by the algorithm when training the BP model. Overall, Fig. 4 shows that the algorithm designed in this paper passes seven iterations to reduce the mean square error to within the allowable range to achieve the ideal training results required by its BP neural network model. 


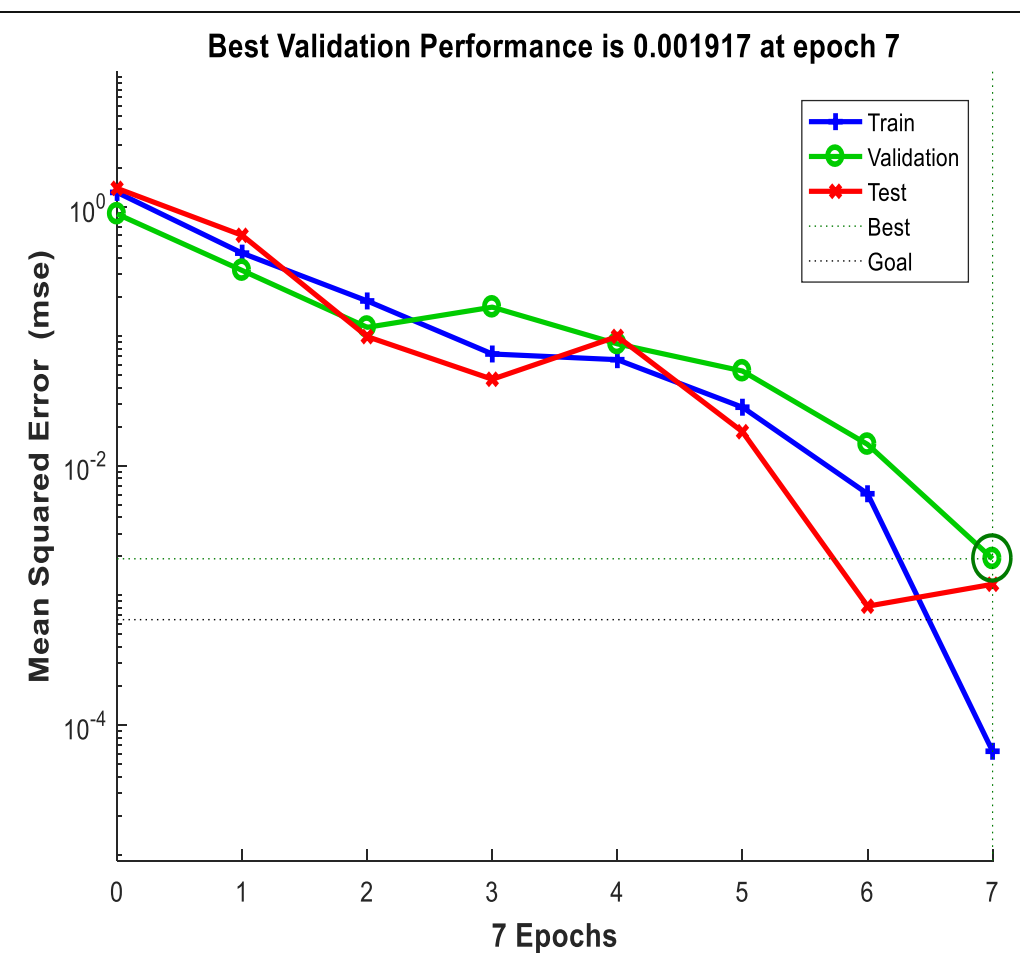

Fig. 4 BP neural network training error curve

The three lines shown in Fig. 5 represent the error surface gradient corresponding to the BP neural network model of the proposed algorithm in the data training process, the momentum parameters of the "trainlm" training function, and the sample data verification detection. According to the data shown in the Fig. 5, the BP neural network model corresponding to the algorithm in this paper has the error surface gradient and the momentum parameter of the "trainlm" training function has reached the expected requirements when training its network. The data verification test also stops in seventh
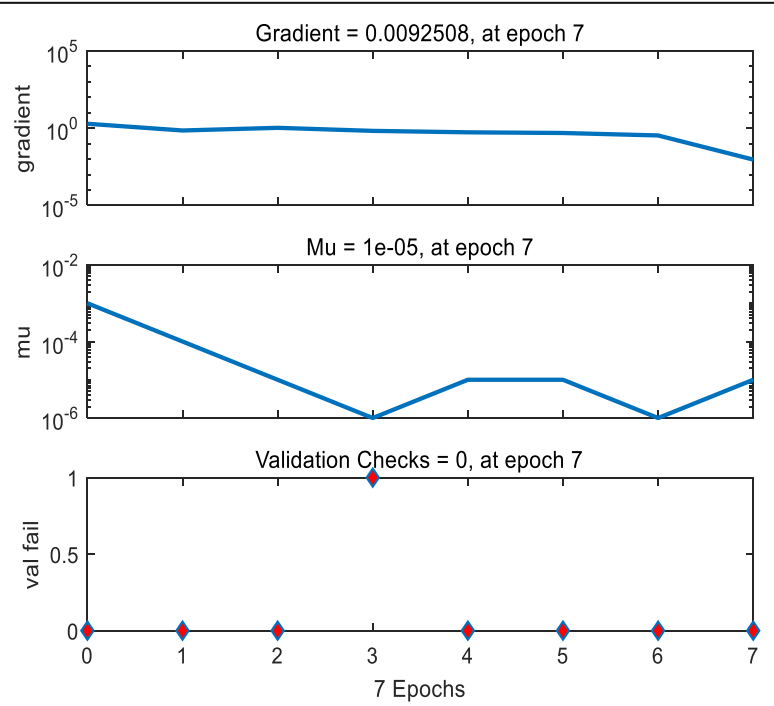

Fig. 5 BP neural network training state curve 

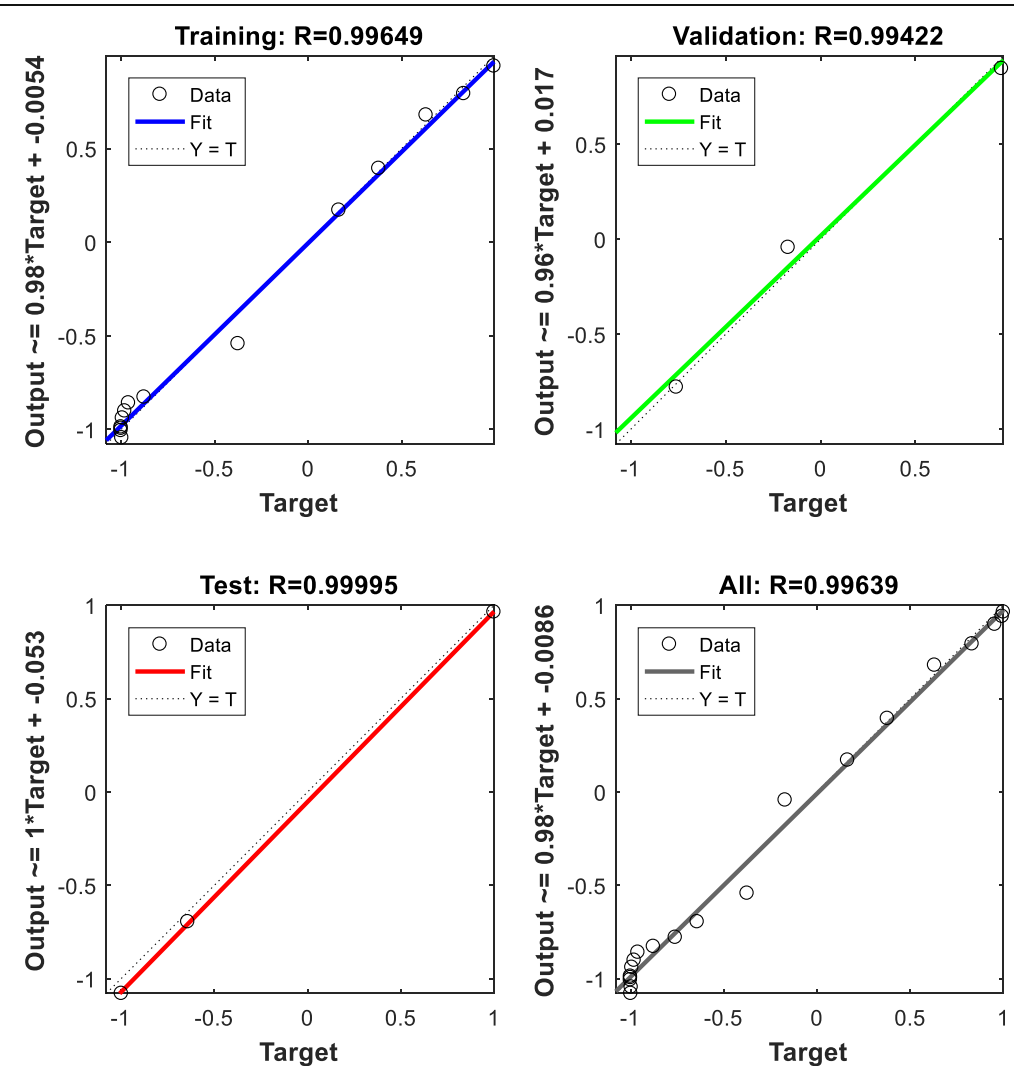

Fig. 6 Regression analysis curve of BP neural network data

steps, indicating that after seven consecutive tests, the error range has stabilized and the system will stop training to prevent over-learning. Therefore, Fig. 5 shows that the training state of the BP neural network is generally in line with expectations.

Figure 6 divides the training data into three parts, namely training, validation, and test. Only training data are used for training, and the other two parts of the data are not used for training. When the training is in progress, since the network is trained based on these data, the error between the target data and the training data will become smaller and smaller, and the error between the validation and validation targets will also become smaller at the beginning, but with as the number of training increases, the error of test continues to decrease, and the error of validation will increase. When the error of validation continues to rise to the number of times the user has set, the training stops (because there is a tendency to overfit). The abscissa of Fig. 6 is the target value set by the user, and the ordinate is the output of the BP neural network. Therefore, in general, the significance of Fig. 6 is to realize the regression analysis of the data. It can be seen from the Fig. 6 that the curves of the four images and the training data are basically on the diagonal, thus achieving a good data fitting, indicating the algorithm of the algorithm. The neural network model achieves an ideal prediction accuracy.

In summary, as can be seen from Figs. 4, 5, and 6, the BP neural network model established by the relevant parameters of the UMTS network achieves the expected error threshold through seven repetitions of learning, thereby completing the data learning, and the same reasoning the corresponding neural network models established 
by the relevant parameters of the four types of networks, GPRS, WLAN, 4G, and 5G, also reach the expected error standard set before data training after undergoing relevant repeated learning. Therefore, in these five types of networks, the results of the data learning and testing of their respective neural network models are shown below.

From the five simulation comparison diagrams given above, it can be seen that after BP neural network data learning, training, and comparison between errors, the average training error of Fig. 7 is $0.61 \%$, and the average training error of Fig. 8 is $0.25 \%$. The average training error of Fig. 9 is $0.50 \%$, the average training error of Fig. 10 is $0.20 \%$, and the average training error of Fig. 11 is $0.21 \%$, so its corresponding UMTS, GPRS, WLAN, 4G, and 5G network prediction download rate and the actual download rate are basically the same and does not exceed the upper limit of $1.00 \%$ of the training error set by the algorithm. Therefore, the prediction data of the BP neural network model composed of these five types of networks can be considered to meet the relevant error requirements. That is, the predicted network download rate obtained under the heterogeneous converged environment composed of these five types of networks is reliable.

Figure 12 shows the results of running the network selection handover strategy for these five types of networks using the vertical handover algorithm designed in this paper on the MATLAB simulation platform.

It can be seen from the above simulation results that the vertical handover algorithm designed in this paper uses the network download rate as the final decision factor to judge the advantages and disadvantages of the five types of networks in the heterogeneous integrated environment, so that the terminal can be in the current environment and switch to the network with the best performance. The simulation results in Fig. 12

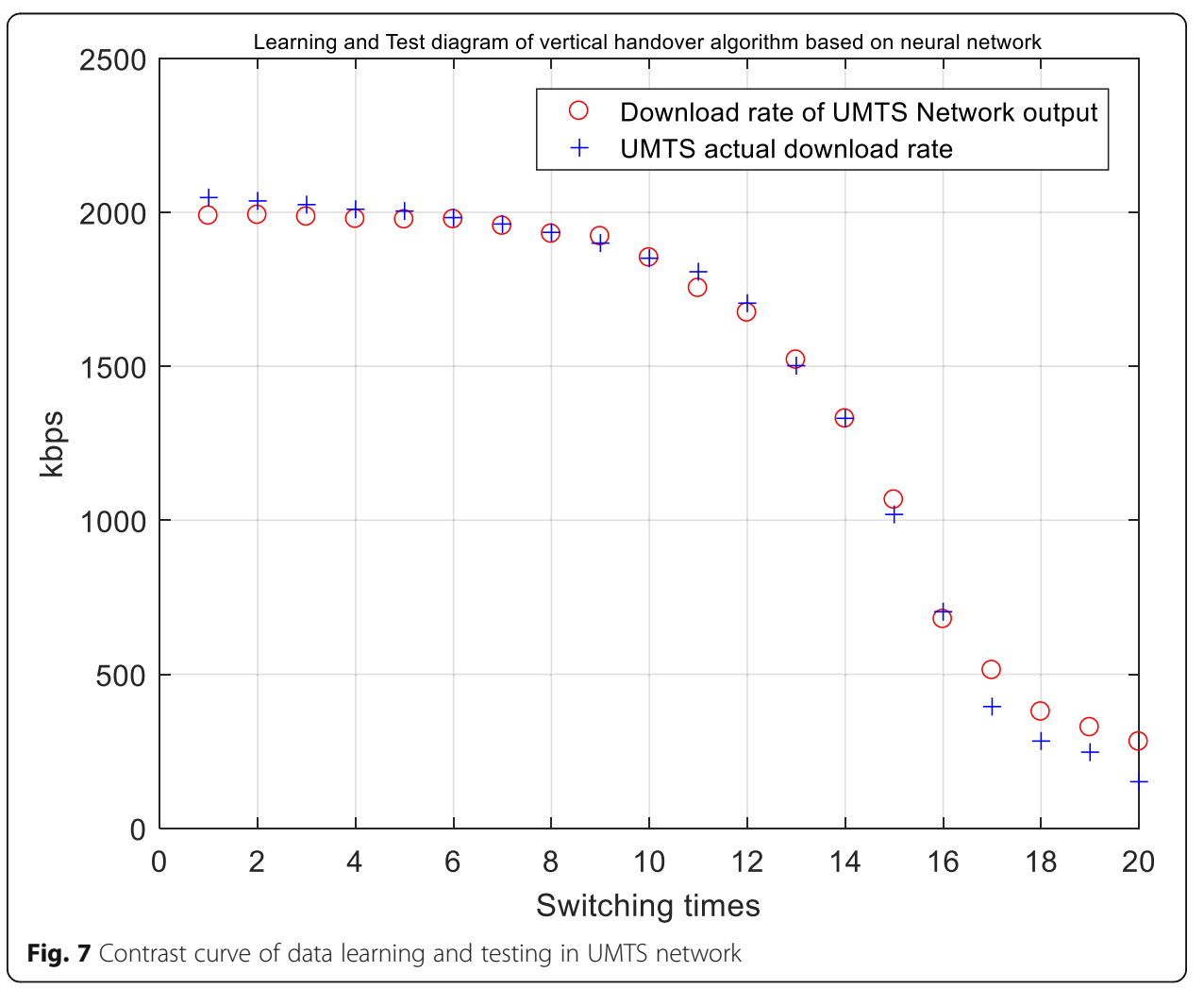




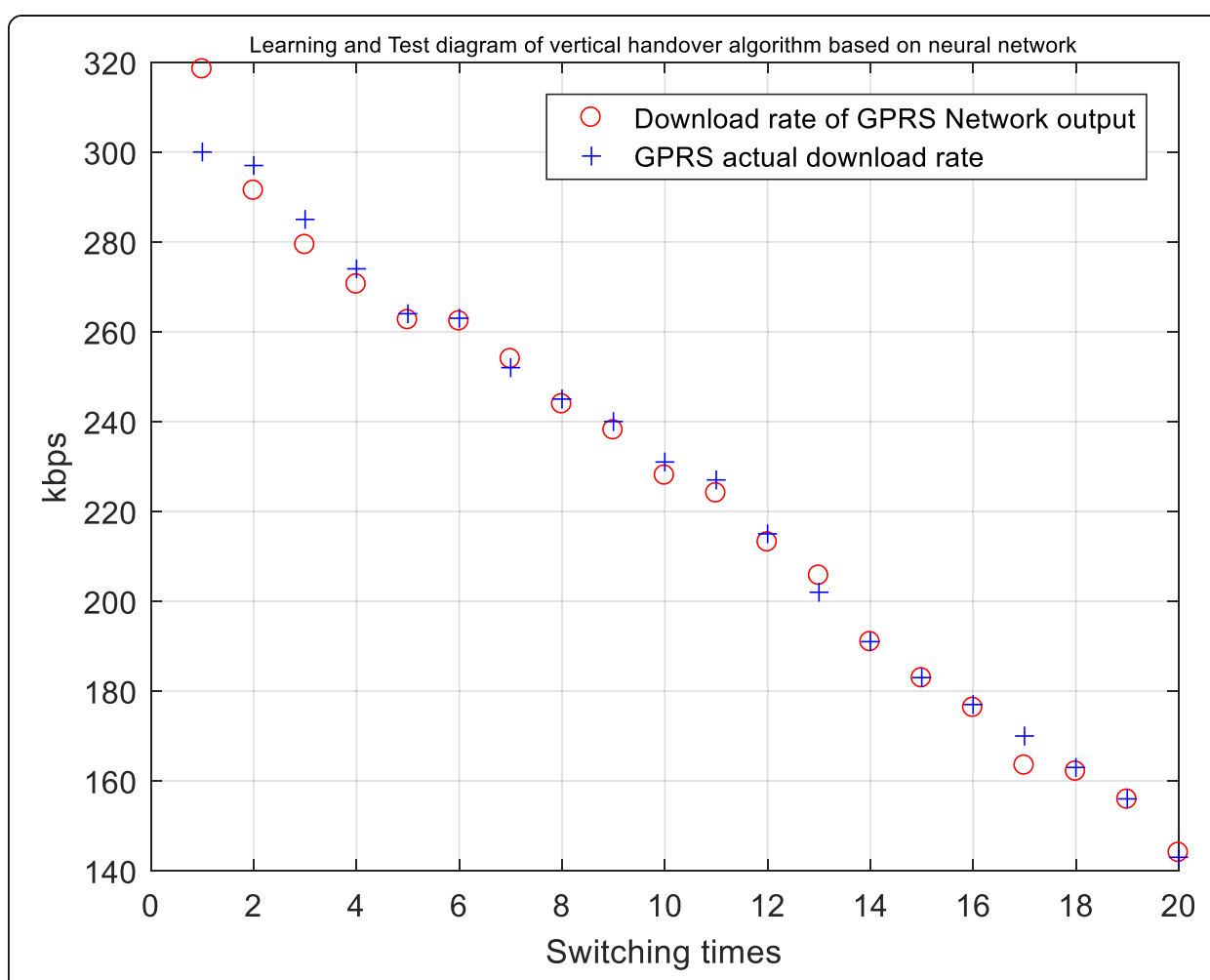

Fig. 8 Contrast curve of data learning and testing in GPRS network

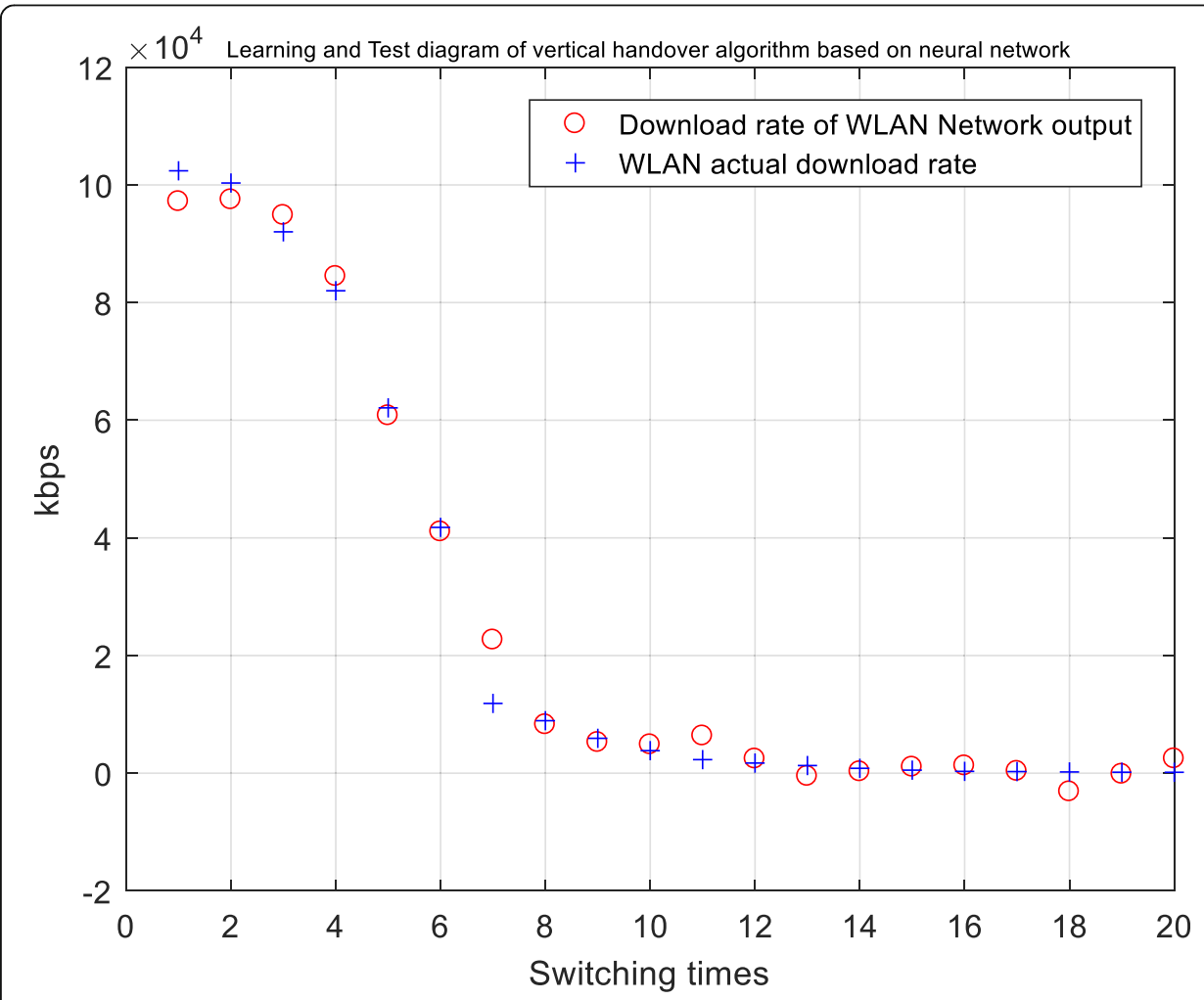

Fig. 9 Contrast curve of data learning and testing in WLAN network 


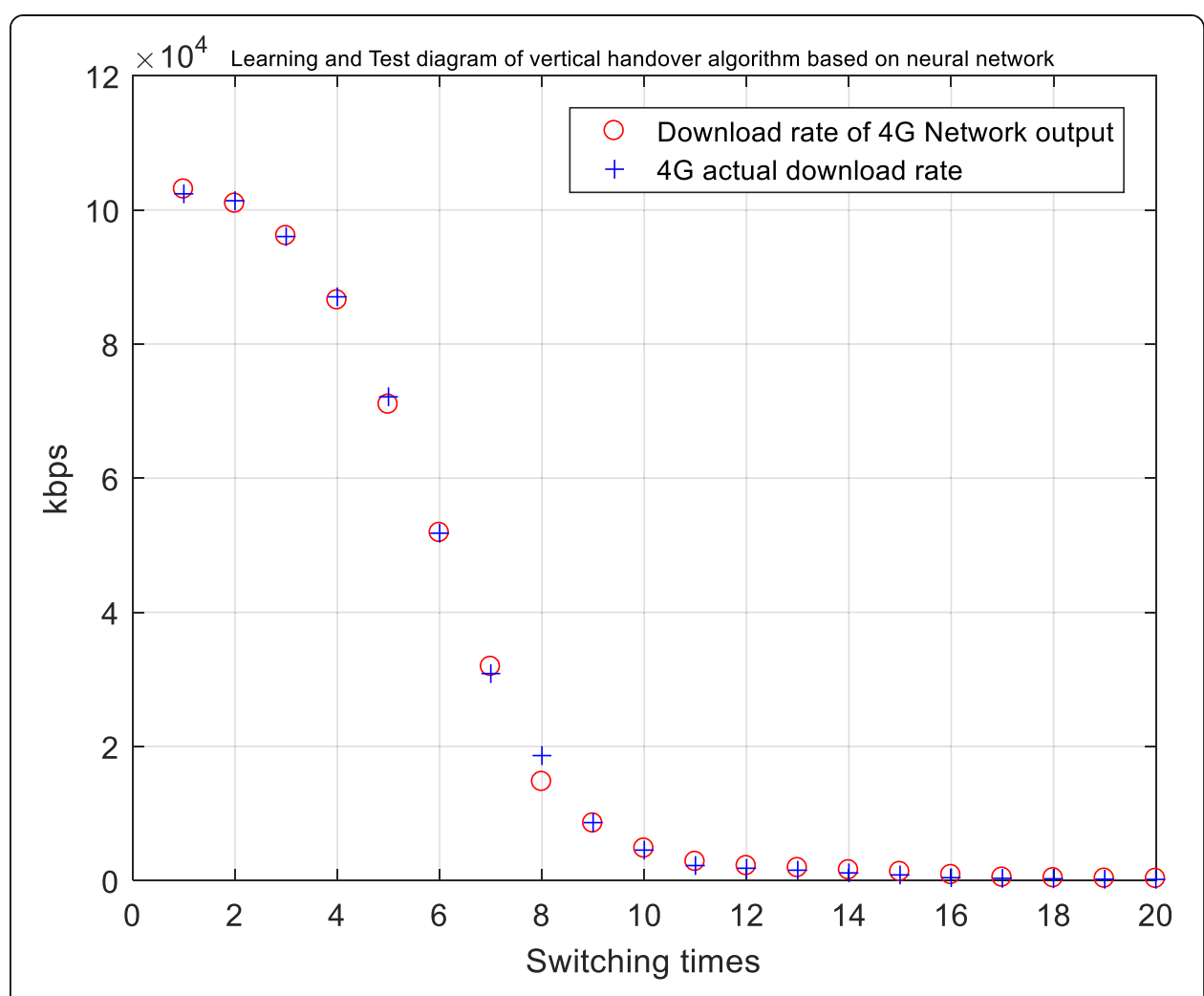

Fig. 10 Contrast curve of data learning and testing in $4 \mathrm{G}$ network

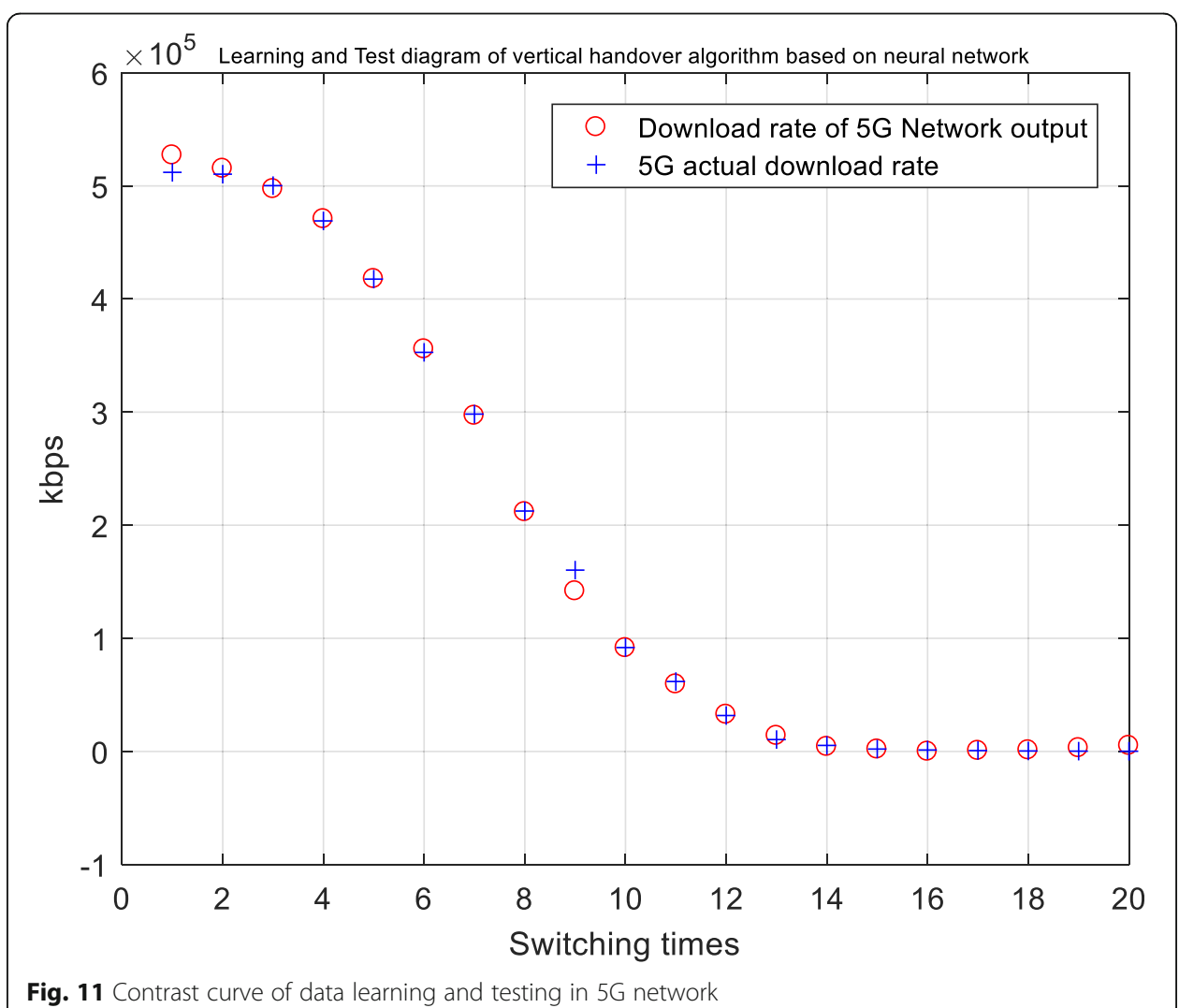




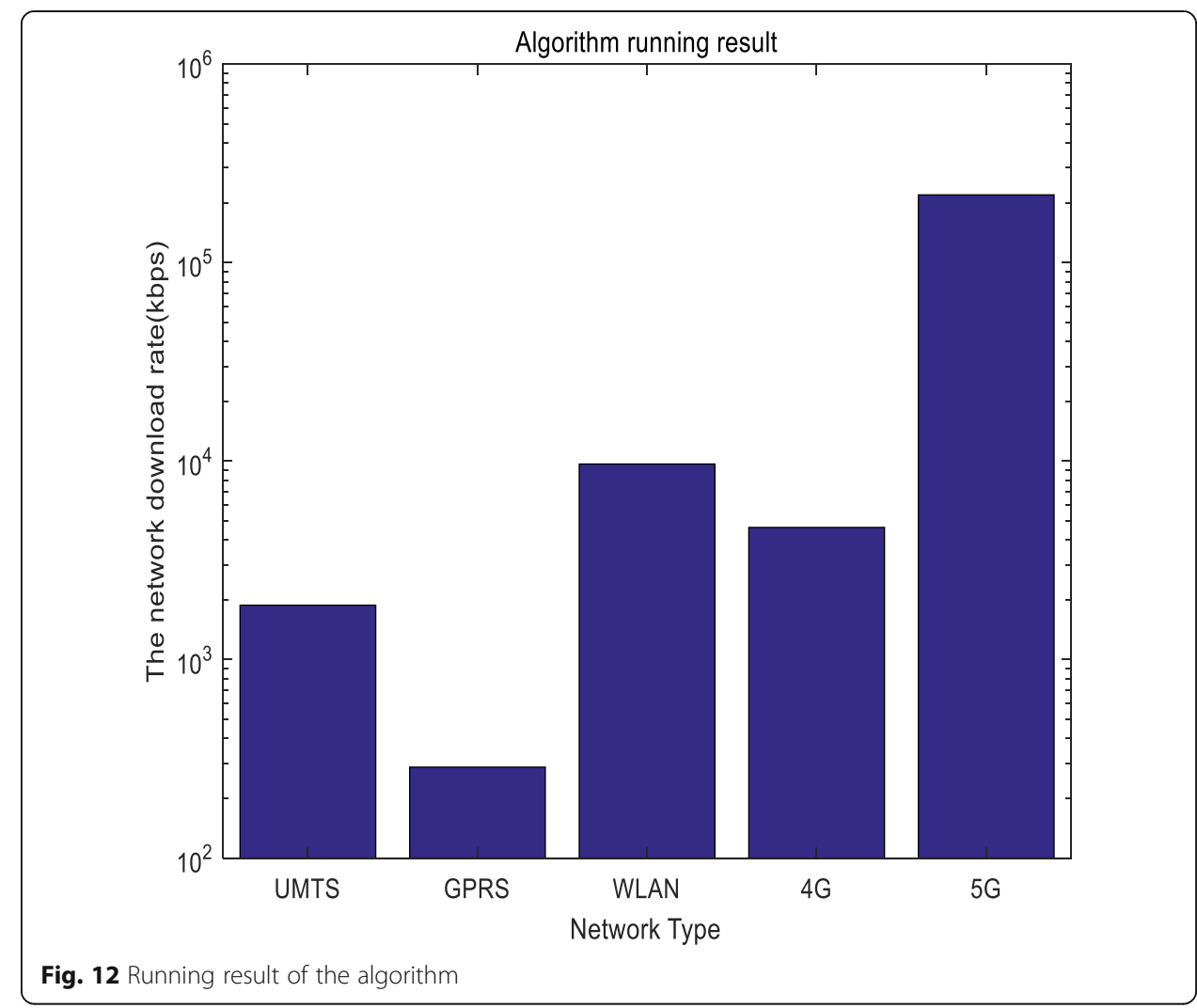

shows that when the algorithm detects the current network environment, the user's mobile rate is $5.1 \mathrm{~m} / \mathrm{s}$, the maximum transmission rate is $930 \mathrm{kbps}$, the minimum transmission delay is $39 \mathrm{~ms}$, the SINR is 13.1 , the bit error rate is $7.1 \times 10^{-6}$, and the packet loss rate is 0.011 , it is predicted that the network download rates of these five types of networks at this moment are $1.8778 \mathrm{Mbps}, 287.0669 \mathrm{Kbps}, 9.6537 \mathrm{Mbps}$, 4.6285 Mbps, and $0.21892 \mathrm{Gbps}$, respectively, and numerical comparison is made. Finally, the $5 \mathrm{G}$ network is obtained and the performance of $5 \mathrm{G}$ is optimal at the moment, thus handover the network selection to the 5G network.

In the field of using intelligent methods to design network vertical handover algorithms, fuzzy logic theory is also widely used. Figure 13 shows the performance comparison between the vertical handover algorithm designed and constructed by the BP neural network framework used in this paper and the vertical handover algorithm designed by the relatively popular fuzzy logic theory. So, a simulation performance comparison of the vertical handover algorithms is constructed. In addition, the comparison results of the handover success rate between the vertical handover algorithm design of the paper and the vertical handover algorithm based on fuzzy logic theory are clearly shown in Fig. 13.

The specific content reflected in Fig. 13 is reflected in the vertical handover algorithm based on the fuzzy logic theory existing in the related references such as the current reference [3], the reference [7], and the reference [17]. The algorithm performs comparative analysis and sets up the same heterogeneous converged network environment on the MATLAB simulation platform, so that the two algorithms respectively perform 20 vertical handover decisions in the same network environment, and the 5G network 


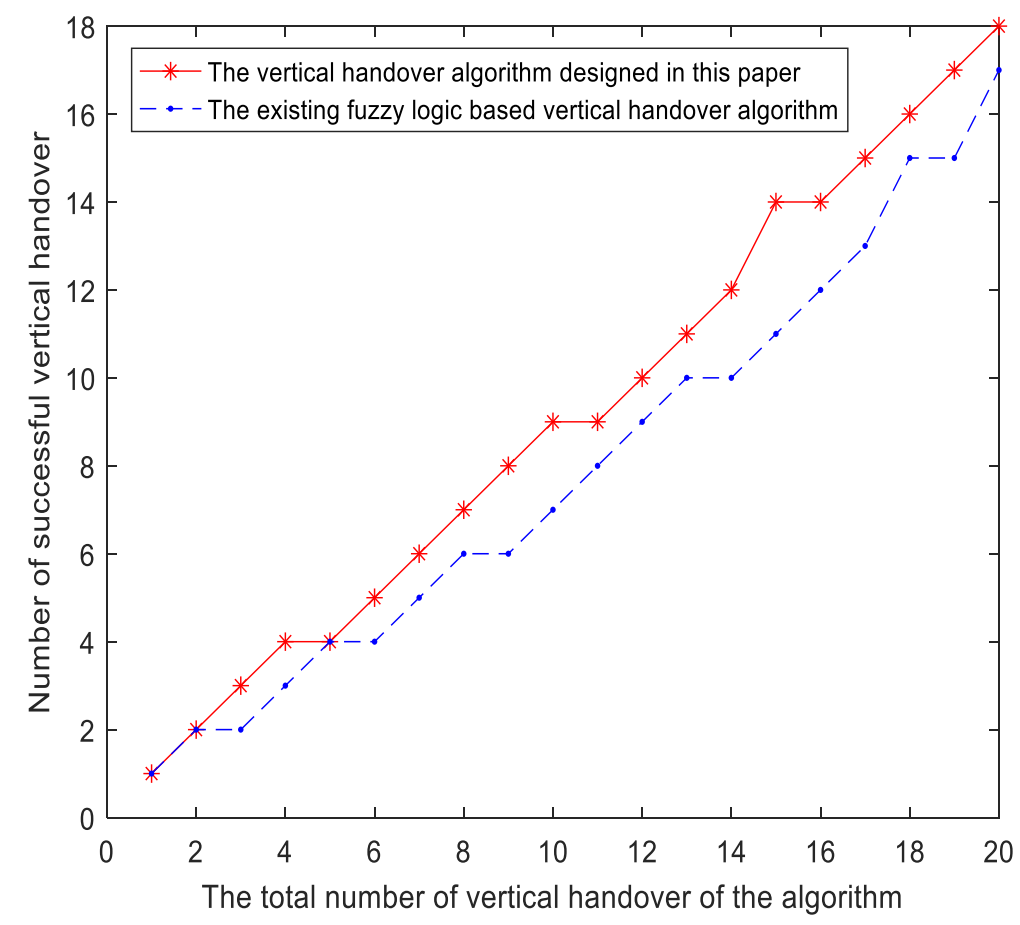

Fig. 13 Comparison results of different kinds of algorithms

is in the set network environment. The downloading rate is the largest and the performance is the precondition. Observe the handover decision of the two types of vertical handover algorithms, that is, select the $5 \mathrm{G}$ network as the success number of the network to be switched, and finally, the vertical handover algorithm designed in this paper is within the error tolerance. The average success of the algorithm is 18 times, that is, the average value of the handover success rate reaches $90 \%$, and the vertical handover algorithm based on fuzzy logic theory that participates in the comparison has an average of 17 handover successes, that is, the average value of the handover success rate reaches $85 \%$. It can be seen that under the condition that there are many types of heterogeneous wireless networks participating in the integrated and the handover environment is more complicated, the handover performance of the vertical algorithm designed in this paper is relatively better than the vertical handover algorithm based on fuzzy logic theory mentioned in the related the reference [3], the reference [7], and the reference [17].

In terms of the user's experience on the wireless network, the download rate of the network is the most important indicator that affects the user experience, and it is also the most significant manifestation of the robustness and other performances. Therefore, we use the network download rate as the final prediction standard for the network selection strategy.

\section{Conclusions}

The vertical handover algorithm designed in this paper introduces the BP neural network model theory and integrates the theory into its running process, thus completing the three-layer BP neural network based on UMTS, GPRS, WLAN, 4G, and 5G 
networks. In the same way, there are also many references presenting a vertical switching algorithm based on neural network, which is similar to the algorithm designed in this paper, and its performance is the same as or even slightly better than the algorithm in this paper in terms of handover success rate, but the vertical handover algorithm based on neural network that has been proposed is generally used in the occasions where there are few types of wireless networks that constitute heterogeneous integrated networks, which makes most of the existing neural network-based vertical handover algorithms contain candidate network types that are not comprehensive and have limited applicability. Taking reference [11] as an example, although it proposes an adaptive vertical handover algorithm based on artificial neural network and the performance is similar to the algorithm designed in this paper, the algorithm designed in reference [11] only selects two wireless networks of LTE and WLAN networks as candidate networks. Obviously, 5G networks with generally better performance than LTE and WLAN are not taken into account. Therefore, compared with the existing algorithms that also use neural networks to perform vertical switching strategies, the algorithm designed in this paper considers the types of related candidate networks that are more comprehensive and the results obtained are more accurate and correct.

To run the algorithm designed in this paper, first, the BP neural network model needs to be constructed, and then the theoretical values of the six major types of network environment attributes such as maximum transmission rate, minimum delay, SINR, bit error rate, user moving speed, and packet loss rate are combined for data learning and network training. Through the actual values of the environmental attribute are input, the network download rate value is used as the final performance measurement factor of the five major networks to predict the data, thus achieving the process of screening the optimal network to complete the handover decision, and finally successfully achieved on the MATLAB platform. The user is allowed to obtain the correct vertical handover simulation result within the scope of the theoretical error allowed in the integrated environment of the above five types of heterogeneous networks. However, the vertical handover algorithm studied in this paper is still in the simulation research stage, but only achieved certain research results in the simulation research stage, therefore in the future research work related to "BP neural network" and "vertical handover." Such algorithms need to be combined with hardware circuits and applied to the areas of the five integrated wireless networks that have similar network performance, so that the algorithm can more efficiently implement vertical handover decisions, then further adjust and improve its operation process during the hardware test in order to improve its practicality in relevant engineering applications.

Finally, based on the performance of the handover success rate measurement algorithm, the performance of the vertical handover algorithm designed in this paper is compared with the performance of other representative vertical handover algorithms. The conclusion that the handover success rate of the vertical handover algorithm designed in this paper is up to $90 \%$ is obtained, and it provides a reference for other research in related fields. 
WiMAX: World Interoperability for Microwave Access; IEEE: Institute of Electrical and Electronics Engineers; WLAN_UMTS: Wireless Local Area Network and Universal Mobile Telephone System

\section{Acknowledgements}

Not applicable.

\section{Authors' contributions}

Conceived and designed the experiments: XNT, GC and HYS. Performed the experiments: XNT. Analyzed the data: XNT and GC. Wrote the paper: XNT, GC and HYS. All authors have contributed to this research work and read and approved the final manuscript.

\section{Authors' information}

Xiaonan Tan received the B.S. degree in communication engineering from Shandong University of Science and Technology, Qingdao, China, in 2018. Now, he is an academic master student in the College of Electronic and Information Engineering of Shandong University of Science and Technology. His research interests are in Internet of things, vertical handover algorithms, neural networks, MPPT, and communication and information system. Geng Chen received the B.S. degree in electronic information engineering and the M.S. degree in communication and information system from Shandong University of Science and Technology, Qingdao, China, in 2007 and 2010, respectively, and the Ph.D. degree in information and communications engineering from Southeast University, Nanjing, China, in 2015. He is currently an Associate Professor at the College of Electronic and Information Engineering, Shandong University of Science and Technology, Qingdao, China. His current research interests are in the areas of heterogeneous networks, ubiquitous networks and software-defined mobile networks, with emphasis on wireless resource management and optimization algorithms, and precoding algorithms in large-scale MIMO.

Hongyu Sun received his Ph.D. degree in Computer Science from Tongji University in 2012. Now, he is an associate professor in the College of Electronic and Information Engineering of Shandong University of Science and Technology. His research interests are in wireless communication theory and advanced digital signal processing system.

\section{Funding}

This work was supported by the National Natural Science Foundation of China under Grant No. 61701284, the China Postdoctoral Science Foundation under Grant No. 2016M602165, the Innovative Research Foundation of Qingdao under Grant No. 19-6-2-1-cg, the Opening Project Fund of State Key Laboratory of Mining Disaster Prevention and Control Co-founded by Shandong Province and the Ministry of Science and Technology under Grant No. MDPC201715, the Key Research and Development project of Shandong Province under Grant No. 2018GHY115022, the Shandong Natural Science Foundation under Grant No. ZR2017MF070, the Qingdao Postdoctoral Application Research Project under Grant No. 2016113, and the Science and Technology Support Plan of Youth Innovation Team of Shandong higher School under Grant No. 2019KJN024.

\section{Availability of data and materials}

Not applicable.

\section{Competing interests}

The authors declare that they have no competing interests.

\section{Author details}

${ }^{1}$ College of Electronic and Information Engineering, Shandong University of Science and Technology, Qingdao 266590, China. ${ }^{2}$ College of Electrical Engineering and Automation, Shandong University of Science and Technology, Qingdao 266590, China.

Received: 30 May 2020 Accepted: 1 October 2020

Published online: 16 October 2020

\section{References}

1. L. Shufei, Z. Yuexia, F. Bo, et al., Multi-attribute vertical handover decision-making algorithm in a hybrid VLC-Femto system. IEEE Commun. Lett. 21(7), 1521-1524 (2017)

2. C. Jiamei, W. Yao, Y. Li, et al., QoE-aware intelligent vertical handover scheme over heterogeneous wireless access networks. IEEE Access. 6, 38285-38293 (2018)

3. R. Gongye, Q. Hua, J. Zhao, Decision-making algorithm for vertical handover based on multi-terminal cooperation based on fuzzy logic. J. Commun. 35(9), 67-78 (2014)

4. E. Obayiuwana, O.E. Falowo, Network selection in heterogeneous wireless networks using multi-criteria decision-making algorithms: a review. Wirel. Netw 23(8), 2617-2649 (2017)

5. A. Chinnappan, R. Balasubramanian, Complexity-consistency trade-off in multi-attribute decision making for vertical handover in heterogeneous wireless networks. IET Networks 5(1), 13-21 (2016)

6. P. Dinesh, H.K. Beom, P. Jae-Young, et al., Maximizing network utilization in IEEE 802.21 assisted vertical handover over wireless heterogeneous networks. Journal of information processing systems 14(3), 771-789 (2018)

7. X. Liu, L. Jiang, C. He, A Pre-decision aided vertical handover algorithm based on fuzzy logic. Electr. J. 35(10), 1889-1993 (2007)

8. Suresha V., and Shankaraiah: Multi attribute vertical handover algorithm for heterogeneous networks. International Journal of Advances in Computer and Electronics Engineering., vol.2, no.3,pp.22-27(2017)

9. A.M. Miyim, M. Ismail, R. Nordin, Performance Analysis of Multi-level Vertical Handover in Wireless Heterogeneous Networks. Wirel. Pers. Commun. 95(2), 1109-1130 (2017) 
10. G. Shidrokh, H.W. Haslina, A.M. Hossein, et al., MDP-based network selection scheme by genetic algorithm and simulated annealing for vertical-handover in heterogeneous wireless networks. Wirel. Pers. Commun. 92(2), 399-436 (2017)

11. Bin Ma., Shangru Li., and Xianzhong Xie.:Adaptive vertical handover algorithm based on artificial neural network in heterogeneous wireless networks. Journal of Electronics and Information., vol.41,no.0, pp.1-7(2019)

12. J.A. Huertas, Y. Donoso, Multi-objective tabu search to balance multihoming loads in heterogeneous wireless networks. International Journal of Computers Communications \& Control 13(6), 956-971 (2018)

13. Chandra I., Prabha K. Helen, amd Sivakumar N.: Optimization of QoS parameters using scheduling techniques in heterogeneous network. Pers. Ubiquit. Comput.., vol.22, no.5-6, pp.943-950,(2018)

14. Baojun LI.: Research on vertical handoff algorithm for heterogeneous converged networks. M.S.thesis, Harbin Engineering Univ., Harbin, China(2014)

15. Almutairi Ali F., Hamed Mohannad., and Landolsi Mohamed Adnan. et al.: A genetic algorithm approach for multiattribute vertical handover decision making in wireless networks. Telecommun. Syst.., vol.68, no.2, pp.151-161(2018)

16. Hewei Yu., and Biao Zhang.: A heterogeneous network selection algorithm based on network attribute and user preference. AD hoc Networks., vol.72, pp. 68-80(2018)

17. X. Qi, J. Liang, N. Xiao, et al., Research on heterogeneous network selection algorithm based on fuzzy multi-attribute comprehensive weight. Modern Defense Technology 41(6), 76-82 (2013)

18. O. Nouri, B. Imen, A. Gharsallah, et al., Smart mobility management in 5 G heterogeneous networks. IET Networks. 7(3), 119-128 (2018)

19. Shichen Li.: Design of several kinds of neural network stability analysis simulation system based on MATLAB. M.S.thesis Chinese Marine Univ., Qingdao, China(2013)

20. Shidrokh Goudarzi., Wan Haslina Hassan., and Mohammad Hossein Anisi.: Intelligent technique for seamless vertical handover in vehicular networks. Mobile Networks \& Applications.,vol.23, no.6, pp.1462-1477(2018)

21. P. Cunhua, Z. Huiling, N.J. Gomes, et al., Joint Precoding and RRH Selection for User-Centric Green MIMO C-RAN. IEEE Trans. Wirel. Commun. 16(5), 2891-2906 (2017)

22. Wang Li.: New Service Application and Key Technology of 5th Generation Mobile Communication Network. Message Communication., no.8, pp.243-245(2018)

23. Jyoti Madaan., and Indu Kashyap.: An Overview of Vertical handover Decision Algorithm. International Journal of Computer Applications., vol.111, no.3, pp.10-15(2015)

24. Huawei Li.: Application of $5 \mathrm{G}$ technology in mobile communication network. Computer Products and Circulation., no.5, pp.72(2018)

25. P. Cunhua, E. Maged, J. Wang, et al., User-centric C-RAN architecture for ultra-dense $5 \mathrm{G}$ networks: challenges and methodologies. IEEE Commun. Mag. 56(6), 14-20 (2018)

26. Siyu Tao., Hongyi Yu., Qing Li. et al.: Performance analysis of user association strategy based on power-domain nonorthogonal multiple access in visible light communication multi-cell networks. EURASIP Journal on Wireless Communications and Networking volume 2020, 80 (2020). https://doi.org/https://doi.org/10.1186/s13638-020-01688-3

27. Marwan Alakhras., Mourad Oussalah., Mousa Hussein.: A survey of fuzzy logic in wireless localization. EURASIP J. Wirel. Commun. Netw. volume 2020, 89 (2020). https://doi.org/https://doi.org/10.1186/s13638-020-01703-7

28. R. Shinkuma, T. Nishio, Y. Inagaki, et al., Data assessment and prioritization in mobile networks for real-time prediction of spatial information using machine learning. EURASIP Journal on Wireless Communications and Networking volume 2020, 92 (2020) https://doi.org/https://doi.org/10.1186/s13638-020-01709-1

29. Qing Yang, Shijue Zheng, Ming Liu. et al.: Research on Wi-Fi indoor positioning in a smart exhibition hall based on received signal strength indication. EURASIP Journal on Wireless Communications and Networking volume 2019, 275 (2019). https://doi.org/https://doi.org/10.1186/s13638-019-1601-3

30. Yansong Liu, Li Zhu: A new intrusion detection and alarm correlation technology based on neural network. EURASIP Journal on Wireless Communications and Networking volume 2019, 109 (2019). https://doi.org/https://doi.org/10.1186/ s13638-019-1419-z

31. Mehdi Aslinezhad, Alireza Malekijavan, Pouya Abbasi: ANN-assisted robust GPS/INS information fusion to bridge GPS outage. EURASIP Journal on Wireless Communications and Networking volume 2019, 129 (2020). https://doi.org/https:// doi.org/10.1186/s13638-020-01747-9

32. Mingxiang Guan, Zhou Wu, Yingjie Cui. et al.: An intelligent wireless channel allocation in HAPS $5 G$ communication system based on reinforcement learning. EURASIP Journal on Wireless Communications and Networking volume 2019, 138 (2019). https://doi.org/https://doi.org/10.1186/s13638-019-1463-8

\section{Publisher's Note}

Springer Nature remains neutral with regard to jurisdictional claims in published maps and institutional affiliations. 